\title{
Effective Transmission Conditions for Domain Decomposition Methods applied to the Time-Harmonic Curl-Curl Maxwell's equations
}

\author{
V. Dolean ${ }^{1}$, M.J. Gander ${ }^{2}$, S. Lanteri ${ }^{3}$, J.F. Lee ${ }^{4}$, Z. Peng ${ }^{5}$
}

\begin{abstract}
The time-harmonic Maxwell equations describe the propagation of electromagnetic waves and are therefore fundamental for the simulation of many modern devices we have become used to in everyday life. The numerical solution of these equations is hampered by two fundamental problems: first, in the high frequency regime, very fine meshes need to be used in order to avoid the pollution effect well known for the Helmholtz equation, and second the large scale systems obtained from the vector valued equations in three spatial dimensions need to be solved by iterative methods, since direct factorizations are not feasible any more at that scale. As for the Helmholtz equation, classical iterative methods applied to discretized Maxwell equations have severe convergence problems.

We explain in this paper a family of domain decomposition methods based on well chosen transmission conditions. We show that all transmission conditions proposed so far in the literature, both for the first and second order formulation of Maxwell's equations, can be written and optimized in the common framework of optimized Schwarz methods, independently of the first or second order formulation one uses, and the performance of the corresponding algorithms is identical. We use a decomposition into transverse electric and transverse magnetic fields to describe these algorithms, which greatly simplifies the convergence analysis of the methods. We illustrate the performance of our algorithms with large scale numerical simulations.
\end{abstract}

Keywords: Optimized Schwarz methods, transmission conditions, Maxwell equations

\footnotetext{
${ }^{1}$ University of Strathclyde, Department of Mathematics and Statistics. Victorita.Dolean@strath.ac.uk

${ }^{2}$ Section de Mathématiques, Université de Genève, CP 64, 1211 Genève, martin.gander@math.unige.ch

${ }^{3}$ INRIA Sophia Antipolis-Méditerranée, France, stephane.lanteri@inria.fr

${ }^{4}$ ElectroScience Laboratory, Ohio State University, U.S.A., jinlee@esl.eng.ohio-state.edu

${ }^{5}$ University of New Mexico, U.S.A., pengz@unm.edu
} 


\section{Introduction}

The first order time dependent Maxwell equations are

$$
\varepsilon \frac{\partial \mathcal{E}}{\partial t}-\nabla \times \mathcal{H}+\sigma \mathcal{E}=-\mathcal{J}, \quad \mu \frac{\partial \mathcal{H}}{\partial t}+\nabla \times \mathcal{E}=0
$$

and domain decomposition methods can be directly applied in space time to these equations, see for example [1] for Schwarz waveform relaxation methods. The time-harmonic Maxwell equations are obtained from (1) by assuming that the source is time periodic, $\mathcal{J}(\mathbf{x}, t)=\mathcal{R} e(\mathbf{J}(\mathbf{x}) \exp (i \omega t))$, which implies

$$
\mathcal{E}(\mathbf{x}, t)=\mathcal{R} e(\mathbf{E}(\mathbf{x}) \exp (i \omega t)), \quad \mathcal{H}(\mathbf{x}, t)=\mathcal{R} e(\mathbf{H}(\mathbf{x}) \exp (i \omega t)),
$$

and leads to the first order time-harmonic Maxwell's equations

$$
(i \omega \varepsilon+\sigma) \mathbf{E}-\nabla \times \mathbf{H}=-\mathbf{J}, \quad i \omega \mu \mathbf{H}+\nabla \times \mathbf{E}=\mathbf{0} .
$$

Eliminating $\mathbf{H}$ in this system of equations, we obtain the second order Maxwell equations

$$
\left(-\omega^{2} \varepsilon+i \omega \sigma\right) \mathbf{E}+\nabla \times\left(\frac{1}{\mu} \nabla \times \mathbf{E}\right)=-i \omega \mathbf{J}
$$

Domain decomposition methods have been developed for the first order formulation (2), see [2], 3], 4], [5], 6], and also for the second order formulation (3), see [7, 8 [section 4.7], [9], 10], [1], 12, [13. Both formulations contain the inherent difficulties of the indefinite Helmholtz equation $\left(\Delta+k^{2}\right) u=f$, namely the pollution effect [14, 15] and the difficulties iterative solvers have with such problems 16. The focus of this paper is the iterative solution of discretized Maxwell equations of the form (2) or (3) using domain decomposition techniques. There is also a large body of literature for Maxwell equations with a very different nature: if one discretizes (1) in time using some implicit scheme, on obtains for example for the trapezoidal rule

$$
\begin{aligned}
& \varepsilon \frac{\mathcal{E}^{n+1}-\mathcal{E}^{n}}{\Delta t}-\nabla \times\left(\frac{\mathcal{H}^{n+1}+\mathcal{H}^{n}}{2}\right)+\sigma\left(\frac{\mathcal{E}^{n+1}+\mathcal{E}^{n}}{2}\right)=-\frac{\mathcal{J}^{n+1}+\mathcal{J}^{n}}{2}, \\
& \mu \frac{\mathcal{H}^{n+1}-\mathcal{H}^{n}}{\Delta t}+\nabla \times\left(\frac{\mathcal{E}^{n+1}+\mathcal{E}^{n}}{2}\right)=0
\end{aligned}
$$

and thus has to solve at each time step a different type of Maxwell's equation of the form

$$
(\varepsilon \sqrt{\eta}+\sigma) \mathbf{E}-\nabla \times \mathbf{H}=-\tilde{\mathbf{J}}, \quad \mu \sqrt{\eta} \mathbf{H}+\nabla \times \mathbf{E}=\mathbf{g},
$$

where $(\mathbf{E}, \mathbf{H}):=\left(\mathcal{E}^{n+1}, \mathcal{H}^{n+1}\right), \sqrt{\eta}:=\frac{2}{\Delta t}, \widetilde{\mathbf{J}}:=\frac{\mathcal{J}^{n+1}+\mathcal{J}^{n}}{2}-\sqrt{\eta} \varepsilon \mathcal{E}^{n}+2 \sigma \mathcal{E}^{n}-$ $\nabla \times \mathcal{H}^{n}$, and $\mathbf{g}=\sqrt{\eta} \mu \mathcal{H}^{n}-\nabla \times \mathcal{E}^{n}$. Here as well, one can eliminate $\mathbf{H}$ from the system and obtains the second order formulation

$$
(\varepsilon \sqrt{\eta}+\sigma) \mathbf{E}+\nabla \times\left(\frac{1}{\mu \sqrt{\eta}} \nabla \times \mathbf{E}\right)=-\tilde{\mathbf{J}}-\nabla \times \frac{1}{\mu \sqrt{\eta}} \mathbf{g} .
$$


There are many very good solvers for this type of Maxwell's equation of the first order form (5) or second order form (6), since, as one can see from comparing them with the time-harmonic formulations $(2)$ and $(3)$, they correspond to the positive definite Helmholtz equation ${ }^{1} \eta u-\Delta u=f$, and for such equations, virtually all iterative methods perform well, especially multigrid and domain decomposition. A classical overlapping Schwarz method has for example been proposed and analyzed for (6) in [19, 20, and an optimized Schwarz method for (5) can be found in the second part of [4. FETI type preconditioners have been developed for (6) with jumping coefficients in 21, and for mortar discretizations in 22]. Primal iterative substructuring methods for the same problem have been analyzed in [23, see also 24] for an effective preconditioner for the Schur complement. Efficient multigrid methods for (6) have been proposed in 25], see also [26. In this paper, we are not interested in the Maxwell type problems of the form (5), (6), where many very good solvers exist, but in the time-harmonic case modeled by (2), (3).

Over the last decade, a new class of overlapping Schwarz methods was developed for scalar partial differential equations, namely the optimized Schwarz methods. These methods are based on a classical overlapping domain decomposition, but they use more effective transmission conditions than the classical Dirichlet conditions at the interfaces between subdomains. New transmission conditions were originally proposed for three different reasons. First, to obtain Schwarz algorithms that are convergent without overlap, see [27] for Robin conditions. The second motivation for changing the transmission conditions was to obtain a convergent Schwarz method for the Helmholtz equation, where the classical overlapping Schwarz algorithm is not convergent. As a remedy, approximate radiation conditions were introduced in [28, 29]. The third motivation was that the convergence rate of the classical Schwarz method is rather slow and too strongly dependent on the size of the overlap. For an introduction to optimized Schwarz methods, see [30].

Like the Helmholtz equation, the high frequency time-harmonic Maxwell's equations are difficult to solve by classical iterative methods. Domain decomposition methods are currently the most promising solution techniques for this class of problems. Optimized transmission conditions for the best performance of the Schwarz algorithm in a given class of local transmission conditions were first introduced for the Helmholtz equation in 31, 32. Following the first provably convergent method in 29, various optimized Schwarz methods were developed over the last decade: in [31, 33, 32, 10, 4, 6, the focus was on optimized polynomial approximations of the symbol of the transparent boundary conditions; for rational approximations of Padé type, see 34.

As far as Maxwell's equations are concerned, there are two basic formulations: the first order formulation (2), for which complete optimized results are known [4, 6, and the second order or curl-curl formulation (3), with par-

\footnotetext{
${ }^{1}$ This terminology is becoming more and more common, in order to well distinguish this equation from the original Helmholtz equation studied by Helmholtz in [17, see also [18]
} 
tial optimization results. Applications to real life problems using a Discontinuous Galerkin method can be found in [2, 3, 35. For finite-element based non-overlapping and non-conforming domain decomposition methods for the computation of multiscale electromagnetic radiation and scattering problems we refer to $36,11,12,13$.

\section{Optimized Schwarz algorithms}

We consider the curl-curl problem (3) with zero conductivity $\sigma=0$ in a bounded domain $\Omega$, with boundary conditions on $\partial \Omega$ such that the problem is well posed [37. We further assume that $\mu$ is constant, and therefore obtain

$$
-\tilde{\omega}^{2} \mathbf{E}+\nabla \times \nabla \times \mathbf{E}=-i \tilde{\omega} Z \mathbf{J}
$$

where $\tilde{\omega}:=\omega \sqrt{\mu \varepsilon}$ and $Z:=\sqrt{\frac{\mu}{\varepsilon}}$. A general Schwarz algorithm for two subdomains $\Omega=\Omega_{1} \cup \Omega_{2}$ then solves iteratively for $n=1,2 \ldots$ the subdomain problems

$$
\begin{aligned}
-\tilde{\omega}^{2} \mathbf{E}^{1, n}+\nabla \times\left(\nabla \times \mathbf{E}^{1, n}\right) & =-i \tilde{\omega} Z \mathbf{J} \text { in } \Omega_{1} \\
\mathcal{T}_{\mathbf{n}_{1}}\left(\mathbf{E}^{1, n}\right) & =\mathcal{T}_{\mathbf{n}_{1}}\left(\mathbf{E}^{2, n-1}\right) \text { on } \Gamma_{12}, \\
-\tilde{\omega}^{2} \mathbf{E}^{2, n}+\nabla \times\left(\nabla \times \mathbf{E}^{2, n}\right) & =-i \tilde{\omega} Z \mathbf{J} \text { in } \Omega_{2} \\
\mathcal{T}_{\mathbf{n}_{2}}\left(\mathbf{E}^{2, n}\right) & =\mathcal{T}_{\mathbf{n}_{2}}\left(\mathbf{E}^{1, n-1}\right) \text { on } \Gamma_{21},
\end{aligned}
$$

where $\Gamma_{12}=\partial \Omega_{1} \cap \Omega_{2}, \Gamma_{21}=\partial \Omega_{2} \cap \Omega_{1}$, and $\mathcal{T}_{\mathbf{n}_{j}}$ are transmission conditions, $\mathbf{n}_{j}$ denoting the unit outward normal vector of subdomain $\Omega_{j}$. The classical Schwarz method uses for example the impedance condition

$$
\mathcal{T}_{\mathbf{n}}(\mathbf{E})=(\nabla \times E \times \mathbf{n}) \times \mathbf{n}+i \tilde{\omega} \mathbf{E} \times \mathbf{n},
$$

see [29]. This impedance condition is equivalent to using the condition

$$
\mathcal{T}_{\mathbf{n}}(\mathbf{E})=(\nabla \times E \times \mathbf{n})-i \tilde{\omega} \mathbf{n} \times(\mathbf{E} \times \mathbf{n}),
$$

which is just a rotation by 90 degrees of (9), but is more adapted to variational formulations, see for example 38 .

The transmission conditions in [4 developed for the first order formulation of Maxwell's equations (2), and for which complete optimization results are available, can be written for the curl-curl formulation (7) in the form

$$
\begin{aligned}
\mathcal{T}_{\mathbf{n}}^{1}(\mathbf{E}) & =\left(I+\left(\delta_{1}^{1} \mathcal{S}_{T M}+\delta_{2}^{1} \mathcal{S}_{T E}\right)\right)(\nabla \times \mathbf{E} \times \mathbf{n}) \times \mathbf{n} \\
& +i \tilde{\omega}\left(I-\left(\delta_{3}^{1} \mathcal{S}_{T M}+\delta_{4}^{1} \mathcal{S}_{T E}\right)\right)(\mathbf{E} \times \mathbf{n})
\end{aligned}
$$

Here $\mathcal{S}_{T M}=\nabla_{\tau} \nabla_{\tau} \cdot \mathcal{S}_{T E}=\nabla_{\tau} \times \nabla_{\tau} \times, \tau$ denotes the tangential direction and $\delta_{l}^{1}, l=1,2,3,4$ are parameters that can be chosen in order to obtain fast convergence, see [4] for several optimized choices. A different form of transmission conditions was proposed in [13] and [12],

$$
\begin{aligned}
\mathcal{T}_{\mathbf{n}}^{2}(\mathbf{E}) & =\left(I+\left(\delta_{1}^{2} \mathcal{S}_{T M}+\delta_{2}^{2} \mathcal{S}_{T E}\right)\right)(\mathbf{n} \times \nabla \times \mathbf{E}) \\
& -i \tilde{\omega}\left(I-\left(\delta_{3}^{2} \mathcal{S}_{T M}+\delta_{4}^{2} \mathcal{S}_{T E}\right)\right)(\mathbf{n} \times(\mathbf{E} \times \mathbf{n}))
\end{aligned}
$$


for more details, see [39]. Using the TE-TM decomposition, we show in the next section that the two general forms of transmission conditions $(11)$ and $(12)$ lead apart from a few subtleties to optimized Schwarz methods with the same contraction factor, and therefore the complete optimization results obtained in [4] for the first order formulation (2) can be used for all these families of Schwarz methods for the curl-curl formulation 3 to obtain the fastest methods in each class. Our analysis in the TE-TM decomposition also shows that one can optimize separately for TE and TM modes, which can be of interest in certain situations.

\section{Convergence analysis using the TE-TM decomposition}

In order to show the relationship between the families of transmission conditions (11) and (12), we use Fourier analysis, and thus assume that the coefficients are constant, and the domain on which the original problem is posed is $\Omega=\mathbb{R}^{3}$, in which case we need to impose the Silver-Müller radiation condition $\lim _{r \rightarrow \infty} r(\nabla \times E \times \mathbf{n}+i \tilde{\omega} \mathbf{E})=0$, where $r=|\mathbf{x}|, \mathbf{n}=\mathbf{x} /|\mathbf{x}|$, for the problem to be well-posed [37]. The two subdomains $\Omega_{1}=(-\infty, L) \times \mathbb{R}^{2}$ and $\Omega_{2}=(0, \infty) \times \mathbb{R}^{2}$ we consider are half spaces, and the interfaces are $\Gamma_{12}=\{L\} \times \mathbb{R}^{2}$ and $\Gamma_{21}=\{0\} \times \mathbb{R}^{2}$, the overlap size being $L \geq 0$. Let the Fourier transform in $y$ and $z$ directions be

$$
\hat{\mathbf{E}}(x, \mathbf{k}):=(\mathcal{F} \mathbf{E})(x, \mathbf{k})=\int_{\mathbb{R}^{2}} \mathbf{E}(x, y, z) e^{i\left(k_{y} y+k_{z} z\right)} d y d z,
$$

where we denote by $k_{y}$ and $k_{z}$ the Fourier variables, $\mathbf{k}=\left(k_{y}, k_{z}\right)$ and $|\mathbf{k}|^{2}=$ $k_{y}^{2}+k_{z}^{2}$. We will also use the hat to denote the Fourier symbols of operators. We first compute the local solutions of the homogeneous counterparts of (8), which corresponds to the equation that the error satisfies at each iteration.

Lemma 1 (Local solutions). The local solutions of (8) with $\mathbf{J}=0$ are of the form

$\hat{\mathbf{E}}^{1}=e^{\lambda(x-L)}\left(-\frac{i\left(A_{2} k_{z}+A_{4} k_{y}\right)}{\lambda}, A_{4}, A_{2}\right)^{T}, \hat{\mathbf{E}}^{2}=e^{-\lambda x}\left(\frac{i\left(A_{1} k_{z}+A_{3} k_{y}\right)}{\lambda}, A_{3}, A_{1}\right)^{T}$

where

$$
\lambda=\sqrt{|\mathbf{k}|^{2}-\tilde{\omega}^{2}}
$$

and the coefficients $A_{1,2,3,4}$ depend in general on $k_{y}, k_{z}$.

Proof. We take a Fourier transform of the curl-curl Maxwell's equations (7) with $\mathbf{J}=0$, and obtain with the notation $\hat{\mathbf{E}}=\left(\hat{E}_{x}, \hat{E}_{y}, \hat{E}_{z}\right)$

$$
\begin{aligned}
& -\tilde{\omega}^{2} \hat{E}_{x}+i k_{y} \frac{d \hat{E}_{y}}{d x}+i k_{z} \frac{d \hat{E}_{z}}{d x}+\left(k_{y}^{2}+k_{z}^{2}\right) \hat{E}_{x}=0, \\
& -\tilde{\omega}^{2} \hat{E}_{y}+i k_{y} \frac{d \hat{E}_{x}}{d x}-k_{y} k_{z} \hat{E}_{z}-\frac{d^{2} \hat{E}_{y}}{d x_{x}^{2}}+k_{z}^{2} \hat{E}_{y}=0, \\
& -\tilde{\omega}^{2} \hat{E}_{z}+i k_{z} \frac{d \hat{E}_{x}}{d x}-k_{y} k_{z} \hat{E}_{y}-\frac{d^{2} \hat{E}_{z}}{d x^{2}}+k_{y}^{2} \hat{E}_{z}=0 .
\end{aligned}
$$


The general solution of this system of ordinary differential equations is

$$
\begin{aligned}
& \hat{E}_{x}=\frac{i\left(A_{3} k_{y}+A_{1} k_{z}\right) e^{-\lambda x}}{\lambda}-\frac{i\left(A_{4} k_{y}+A_{2} k_{z}\right) e^{\lambda x}}{\lambda}, \\
& \hat{E}_{y}=A_{3} e^{-\lambda x}+A_{4} e^{\lambda x} \\
& \hat{E}_{z}=A_{1} e^{-\lambda x}+A_{2} e^{\lambda x} .
\end{aligned}
$$

Using now the Silver-Müller conditions in both subdomains we obtain (13).

The local solutions in (13) suggest to use a different basis, which we call the TE-TM decomposition.

Lemma 2 (TE-TM decomposition of local solutions). The local solutions from (13) can be re-written as

$$
\hat{\mathbf{E}}^{j}=A_{T M} \hat{\mathbf{E}}^{j, T M}+A_{T E} \hat{\mathbf{E}}^{j, T E}, j=1,2,
$$

where

$$
\begin{gathered}
\hat{\mathbf{E}}^{1, T E}=e^{\lambda(x-L)}\left(0,-\frac{k_{z}}{k_{y}}, 1\right)^{T}, \hat{\mathbf{E}}^{1, T M}=e^{\lambda(x-L)}\left(-\frac{i|\mathbf{k}|^{2}}{k_{y} \lambda}, 1, \frac{k_{z}}{k_{y}}\right)^{T}, \\
\hat{\mathbf{E}}^{2, T E}=e^{-\lambda x}\left(0,-\frac{k_{z}}{k_{y}}, 1\right)^{T}, \hat{\mathbf{E}}^{2, T M}=e^{-\lambda x}\left(\frac{i|\mathbf{k}|^{2}}{k_{y} \lambda}, 1, \frac{k_{z}}{k_{y}}\right)^{T} .
\end{gathered}
$$

Proof. We split the solution in $\Omega_{1}$ from 13 into a combination of solutions verifying $A_{2} k_{z}+A_{4} k_{y}=0, A_{2}, A_{4} \neq 0$ called TE modes, and the orthogonal complement called TM modes. The relation $A_{2} k_{z}+A_{4} k_{y}=0$ implies that $A_{4}=-A_{2} \frac{k_{z}}{k_{y}}$, and therefore, choosing $A_{2}=1$, a basis vector for the TE mode in $\Omega_{1}$ is $\hat{\mathbf{E}}^{1, T E}$ given in 18 . To find the corresponding TM mode, we need to find a vector of the form $\hat{\mathbf{E}}^{1}$ in 13 orthogonal to $\hat{\mathbf{E}}^{1, T E}$. Such a vector is orthogonal to $\hat{\mathbf{E}}^{1, T E}$ if we choose $A_{4}:=1$ and $A_{2}:=\frac{k_{z}}{k_{y}}$ which leads to the vector $\hat{\mathbf{E}}^{1, T M}$ in 18 . The result for subdomain $\Omega_{2}$ is obtained similarly.

The action of the operators involved in the transmission conditions 111 and (12) becomes very simple with the TE-TM decomposition.

Lemma 3 (Action of operators in $\mathcal{T}_{\mathbf{n}}^{1}$ on TE-TM modes). For the normals $\mathbf{n}_{1}=(1,0,0)$ and $\mathbf{n}_{2}=(-1,0,0)$, the operators in $\mathcal{T}_{\mathbf{n}}^{1}$ applied to $T E$ and $T M$ modes satisfy for $j=1,2$ the relations

$$
\begin{aligned}
\left(\hat{\nabla} \times \hat{\mathbf{E}}^{j, T E} \times \mathbf{n}_{j}\right) \times \mathbf{n}_{j} & =\lambda\left(\hat{\mathbf{E}}^{j, T E} \times \mathbf{n}_{j}\right), \\
\left(\hat{\nabla} \times \hat{\mathbf{E}}^{j, T M} \times \mathbf{n}_{j}\right) \times \mathbf{n}_{j} & =-\frac{\tilde{\omega}^{2}}{\lambda}\left(\hat{\mathbf{E}}^{j, T M} \times \mathbf{n}_{j}\right), \\
\hat{\mathcal{S}}_{T E}\left(\left(\hat{\nabla} \times \hat{\mathbf{E}}^{j, T E} \times \mathbf{n}_{j}\right) \times \mathbf{n}_{j}\right) & =\hat{\mathcal{S}}_{T E}\left(\hat{\mathbf{E}}^{j, T E} \times \mathbf{n}_{j}\right)=0, \\
\hat{\mathcal{S}}_{T M}\left(\left(\hat{\nabla} \times \hat{\mathbf{E}}^{j, T M} \times \mathbf{n}_{j}\right) \times \mathbf{n}_{j}\right) & =\hat{\mathcal{S}}_{T M}\left(\hat{\mathbf{E}}^{j, T M} \times \mathbf{n}_{j}\right)=0, \\
\hat{\mathcal{S}}_{T E}\left(\hat{\mathbf{E}}^{j, T M} \times \mathbf{n}_{j}\right) & =|\mathbf{k}|^{2}\left(\hat{\mathbf{E}}^{j, T M} \times \mathbf{n}_{j}\right), \\
\hat{\mathcal{S}}_{T M}\left(\hat{\mathbf{E}}^{j, T E} \times \mathbf{n}_{j}\right) & =-|\mathbf{k}|^{2}\left(\hat{\mathbf{E}}^{j, T E} \times \mathbf{n}_{j}\right) .
\end{aligned}
$$


Proof. We first compute $\hat{\nabla} \times \hat{\mathbf{E}}^{j, T E}$ and $\hat{\nabla} \times \hat{\mathbf{E}}^{j, T M}$,

$$
\begin{gathered}
\hat{\nabla} \times \hat{\mathbf{E}}^{1, T E}=\left[\begin{array}{c}
\frac{i|\mathbf{k}|^{2}}{k_{y}} \\
-\lambda \\
-\lambda \frac{k_{z}}{k_{y}}
\end{array}\right] e^{\lambda(x-L)}, \hat{\nabla} \times \hat{\mathbf{E}}^{1, T M}=\left[\begin{array}{c}
0 \\
\frac{k_{z} \tilde{\omega}^{2}}{k_{y} \lambda} \\
-\frac{\tilde{\omega}^{2}}{\lambda}
\end{array}\right] e^{\lambda(x-L)}, \\
\hat{\nabla} \times \hat{\mathbf{E}}^{2, T E}=\left[\begin{array}{c}
\frac{i|\mathbf{k}|^{2}}{k_{y}} \\
\lambda \\
\lambda \frac{k_{z}}{k_{y}}
\end{array}\right] e^{-\lambda x}, \hat{\nabla} \times \hat{\mathbf{E}}^{2, T M}=\left[\begin{array}{c}
0 \\
-\frac{k_{z} \tilde{\omega}^{2}}{k_{y} \lambda} \\
\frac{\tilde{\omega}^{2}}{\lambda}
\end{array}\right] e^{-\lambda x} .
\end{gathered}
$$

We now use the fact that for any vector field $\mathbf{U}=\left(U_{x}, U_{y}, U_{z}\right)$ we have the relations

$\mathbf{n}_{1} \times \mathbf{U}=\left[\begin{array}{r}0 \\ -U_{z} \\ U_{y}\end{array}\right], \mathbf{n}_{2} \times \mathbf{U}=\left[\begin{array}{r}0 \\ U_{z} \\ -U_{y}\end{array}\right], \mathbf{n}_{j} \times\left(\mathbf{U} \times \mathbf{n}_{j}\right)=\left[\begin{array}{c}0 \\ U_{y} \\ U_{z}\end{array}\right], j=1,2$.

Applying the first two relations of $(23)$ to the quantities $\hat{\mathbf{E}}^{j, T E}$ and $\hat{\mathbf{E}}^{j, T M}$ and the last relation of (23) to their curl, and using the symbols

$$
\hat{\mathcal{S}}_{T E}=\left[\begin{array}{cc}
k_{z}^{2} & -k_{y} k_{z} \\
-k_{y} k_{z} & k_{y}^{2}
\end{array}\right], \quad \hat{\mathcal{S}}_{T M}=\left[\begin{array}{cc}
-k_{y}^{2} & -k_{y} k_{z} \\
-k_{y} k_{z} & -k_{z}^{2}
\end{array}\right],
$$

we find after a short calculation the relations (19), 20, 21).

Lemma 4 (Action of operators in $\mathcal{T}_{\mathbf{n}}^{2}$ on TE-TM modes). Similarly, the operators in $\mathcal{T}_{\mathbf{n}}^{2}$ applied to $T E$ and $T M$ modes satisfy for $j=1,2$ the relations

$$
\begin{gathered}
\mathbf{n}_{j} \times \hat{\nabla} \times \hat{\mathbf{E}}^{j, T E}=-\lambda\left(\mathbf{n}_{j} \times\left(\hat{\mathbf{E}}^{j, T E} \times \mathbf{n}_{j}\right)\right), \\
\mathbf{n}_{j} \times \hat{\nabla} \times \hat{\mathbf{E}}^{j, T M}=\frac{\tilde{\omega}^{2}}{\lambda}\left(\mathbf{n}_{j} \times\left(\hat{\mathbf{E}}^{j, T M} \times \mathbf{n}_{j}\right)\right) . \\
\hat{\mathcal{S}}_{T E}\left(\mathbf{n}_{j} \times \hat{\nabla} \times \hat{\mathbf{E}}^{j, T M}\right)=\hat{\mathcal{S}}_{T E}\left(\mathbf{n}_{j} \times\left(\hat{\mathbf{E}}^{j, T M} \times \mathbf{n}_{j}\right)\right)=0, \\
\hat{\mathcal{S}}_{T M}\left(\mathbf{n}_{j} \times \hat{\nabla} \times \hat{\mathbf{E}}^{j, T E}\right)=\hat{\mathcal{S}}_{T M}\left(\mathbf{n}_{j} \times\left(\hat{\mathbf{E}}^{j, T E} \times \mathbf{n}_{j}\right)\right)=0 . \\
\hat{\mathcal{S}}_{T E}\left(\mathbf{n}_{j} \times\left(\hat{\mathbf{E}}^{j, T E} \times \mathbf{n}_{j}\right)\right)=|\mathbf{k}|^{2}\left(\mathbf{n}_{j} \times\left(\hat{\mathbf{E}}^{j, T E} \times \mathbf{n}_{j}\right)\right), \\
\hat{\mathcal{S}}_{T M}\left(\mathbf{n}_{j} \times\left(\hat{\mathbf{E}}^{j, T M} \times \mathbf{n}_{j}\right)\right)=-|\mathbf{k}|^{2}\left(\mathbf{n}_{j} \times\left(\hat{\mathbf{E}}^{j, T M} \times \mathbf{n}_{j}\right)\right) .
\end{gathered}
$$

Proof. We proceed as in the proof of Lemma 3 except that we apply the last relation of $\sqrt{23}$ to the quantities $\hat{\mathbf{E}}^{j, T E}$ and $\hat{\mathbf{E}}^{j, T M}$ and the first two relations of 23 ) to their curl.

Theorem 1 (Convergence factors). The convergence factor of the Schwarz algorithm (8) with transmission conditions (11) and overlap $L \geq 0$ is given by

$$
\rho_{1}=\left|\frac{\lambda-i \tilde{\omega}}{\lambda+i \tilde{\omega}}\right| \max \left\{\left|\frac{1-(\lambda+i \tilde{\omega})\left(\delta_{1}^{1} \lambda+i \tilde{\omega} \delta_{3}^{1}\right)}{1-(\lambda-i \tilde{\omega})\left(\delta_{1}^{1} \lambda-i \tilde{\omega} \delta_{3}^{1}\right)}\right|,\left|\frac{1-(\lambda+i \tilde{\omega})\left(\lambda \delta_{4}^{1}+i \tilde{\omega} \delta_{2}^{1}\right)}{1-(\lambda-i \tilde{\omega})\left(\lambda \delta_{4}^{1}-i \tilde{\omega} \delta_{2}^{1}\right)}\right|\right\}\left|e^{-\lambda L}\right|
$$


where $\lambda=\sqrt{|\mathbf{k}|^{2}-\tilde{\omega}^{2}}$, see (14), and $\tilde{\omega}=\omega \sqrt{\varepsilon \mu}$. With transmission conditions (12), the convergence factor is

$$
\rho_{2}=\left|\frac{\lambda-i \tilde{\omega}}{\lambda+i \tilde{\omega}}\right| \max \left\{\left|\frac{1+(\lambda+i \tilde{\omega})\left(\delta_{2}^{2} \lambda+i \tilde{\omega} \delta_{4}^{2}\right)}{1+(\lambda-i \tilde{\omega})\left(\delta_{2}^{2} \lambda-i \tilde{\omega} \delta_{4}^{2}\right)}\right|,\left|\frac{1+(\lambda+i \tilde{\omega})\left(\lambda \delta_{3}^{2}+i \tilde{\omega} \delta_{1}^{2}\right.}{1+(\lambda-i \tilde{\omega})\left(\lambda \delta_{3}^{2}-i \tilde{\omega} \delta_{1}^{2}\right)}\right|\right\}\left|e^{-\lambda L}\right| .
$$

Therefore if we choose $\delta_{2}^{2}=-\delta_{1}^{1}, \delta_{4}^{2}=-\delta_{3}^{1}, \delta_{1}^{2}=-\delta_{2}^{1}, \delta_{3}^{2}=-\delta_{4}^{1}$, the two Schwarz algorithms are completely equivalent.

Proof. We first compute the action of the interface operators from 111). We obtain

$$
\begin{aligned}
\hat{\mathcal{T}}_{\mathbf{n}_{j}}^{1}\left(\hat{\mathbf{E}}^{j}\right) & =A_{T E}^{j}\left[\left(1+\left(\delta_{1}^{1} \hat{\mathcal{S}}_{T M}+\delta_{2}^{1} \hat{\mathcal{S}}_{T E}\right)\right)\left(\nabla \times \hat{\mathbf{E}}^{j, T E} \times \mathbf{n}_{j}\right) \times \mathbf{n}_{j}\right. \\
& \left.+i \tilde{\omega}\left(1-\left(\delta_{3}^{1} \hat{\mathcal{S}}_{T M}+\delta_{4}^{1} \hat{\mathcal{S}}_{T E}\right)\right)\left(\hat{\mathbf{E}}^{j, T E} \times \mathbf{n}_{j}\right)\right] \\
& +A_{T M}^{j}\left[\left(1+\left(\delta_{1}^{1} \hat{\mathcal{S}}_{T M}+\delta_{2}^{1} \hat{\mathcal{S}}_{T E}\right)\right)\left(\nabla \times \hat{\mathbf{E}}^{j, T M} \times \mathbf{n}_{j}\right) \times \mathbf{n}_{j}\right. \\
& \left.+i \tilde{\omega}\left(1-\left(\delta_{3}^{1} \hat{\mathcal{S}}_{T M}+\delta_{4}^{1} \hat{\mathcal{S}}_{T E}\right)\right)\left(\hat{\mathbf{E}}^{j, T M} \times \mathbf{n}_{j}\right)\right] .
\end{aligned}
$$

By using now properties 19 and 20 from Lemma 3 this simplifies to

$$
\begin{aligned}
\hat{\mathcal{T}}_{\mathbf{n}_{j}}^{1}\left(\hat{\mathbf{E}}^{j}\right) & =A_{T E}^{j}\left[\left(1+\delta_{1}^{1} \hat{\mathcal{S}}_{T M}\right) \lambda \pm i \tilde{\omega}\left(1-\delta_{3}^{1} \hat{\mathcal{S}}_{T M}\right)\right]\left(\hat{\mathbf{E}}^{j, T E} \times \mathbf{n}_{j}\right) \\
& +A_{T M}^{j}\left[\left(1+\delta_{2}^{1} \hat{\mathcal{S}}_{T E}\right)\left(-\frac{\tilde{\omega}^{2}}{\lambda}\right) \pm i \tilde{\omega}\left(1-\delta_{4}^{1} \hat{\mathcal{S}}_{T E}\right)\right]\left(\hat{\mathbf{E}}^{j, T M} \times \mathbf{n}_{j}\right) .
\end{aligned}
$$

where the upper signs of \pm and $\mp$ correspond to $j=1$ and the lower signs to $j=2$. Using now 21) and the relation $|\mathbf{k}|^{2}=(\lambda-i \tilde{\omega})(\lambda+i \tilde{\omega})$ leads to

$$
\begin{aligned}
\hat{\mathcal{T}}_{\mathbf{n}_{j}}^{1}\left(\hat{\mathbf{E}}^{j}\right) & =(\lambda \pm i \tilde{\omega})\left[A_{T E}^{j}\left(1-(\lambda \mp i \tilde{\omega})\left(\delta_{1}^{1} \lambda \mp i \tilde{\omega} \delta_{3}^{1}\right)\right)\left(\hat{\mathbf{E}}^{j, T E} \times \mathbf{n}_{j}\right)\right. \\
& \left.+A_{T M}^{j}\left( \pm \frac{i \tilde{\omega}}{\lambda}+(\lambda \mp i \tilde{\omega})\left(-\delta_{2}^{1} \frac{\tilde{\omega}^{2}}{\lambda} \mp i \tilde{\omega} \delta_{4}^{1}\right)\right)\left(\hat{\mathbf{E}}^{j, T M} \times \mathbf{n}_{j}\right)\right] \\
& =(\lambda \pm i \tilde{\omega})\left[A_{T E}^{j}\left(1-(\lambda \mp i \tilde{\omega})\left(\delta_{1}^{1} \lambda \mp i \tilde{\omega} \delta_{3}^{1}\right)\right)\left(\begin{array}{c} 
\pm 1 \\
\pm \frac{k_{z}}{k_{y}}
\end{array}\right)\right. \\
& \left.+A_{T M}^{j}\left( \pm \frac{i \tilde{\omega}}{\lambda}+(\lambda \mp i \tilde{\omega})\left(-\delta_{2}^{1} \frac{\tilde{\omega}^{2}}{\lambda} \mp i \tilde{\omega} \delta_{4}^{1}\right)\right)\left(\begin{array}{c} 
\pm \frac{k_{z}}{k_{y}} \\
\mp 1
\end{array}\right)\right],
\end{aligned}
$$

where we used the expressions of the TE and TM modes (18) from Lemma 2 We thus obtain

$$
\hat{\mathcal{T}}_{\mathbf{n}_{j}}^{1}\left(\hat{\mathbf{E}}^{j}\right)=B_{j}\left[\begin{array}{c}
A_{T E}^{j} \\
A_{T M}^{j}
\end{array}\right]
$$

and the matrices

$$
B_{j}:=\left[\begin{array}{cc} 
\pm\left(1-(\lambda \mp i \tilde{\omega})\left(\delta_{1}^{1} \lambda \mp i \tilde{\omega} \delta_{3}^{1}\right)\right) & \pm \frac{k_{z}}{k_{y}}\left( \pm \frac{i \tilde{\omega}}{\lambda}+(\lambda \mp i \tilde{\omega})\left(-\delta_{2}^{1} \frac{\tilde{\omega}^{2}}{\lambda} \mp i \tilde{\omega} \delta_{4}^{1}\right)\right) \\
\pm \frac{k_{z}}{k_{y}}\left(1-(\lambda \mp i \tilde{\omega})\left(\delta_{1}^{1} \lambda \mp i \tilde{\omega} \delta_{3}^{1}\right)\right) & \mp\left(1-(\lambda \mp i \tilde{\omega})\left(\delta_{1}^{1} \lambda \mp i \tilde{\omega} \delta_{3}^{1}\right)\right.
\end{array}\right] .
$$

can be re-written as

$$
B_{1}=(\lambda+i \tilde{\omega})\left[\begin{array}{cc}
\alpha & \frac{k_{z}}{k_{y}} \beta \\
\frac{k_{z}}{k_{y}} \alpha & -\beta
\end{array}\right], B_{2}=(\lambda-i \tilde{\omega})\left[\begin{array}{cc}
-\tilde{\alpha} & -\frac{k_{z}}{k_{y}} \tilde{\beta} \\
-\frac{k_{z}}{k_{y}} \tilde{\alpha} & \tilde{\beta}
\end{array}\right],
$$


with

$$
\begin{aligned}
& \alpha=1-(\lambda-i \tilde{\omega})\left(\delta_{1}^{1} \lambda-i \tilde{\omega} \delta_{3}^{1}\right) \\
& \tilde{\alpha}=1-(\lambda+i \tilde{\omega})\left(\delta_{1}^{1} \lambda+i \tilde{\omega} \delta_{3}^{1}\right) \\
& \beta=\frac{i \tilde{\omega}}{\lambda}\left(1-(\lambda-i \tilde{\omega})\left(\lambda \delta_{4}^{1}-i \tilde{\omega} \delta_{2}^{1}\right)\right) \\
& \tilde{\beta}=-\frac{i \tilde{\omega}}{\lambda}\left(1-(\lambda+i \tilde{\omega})\left(\lambda \delta_{4}^{1}+i \tilde{\omega} \delta_{2}^{1}\right)\right)
\end{aligned}
$$

Therefore, the Schwarz iteration (8) with transmission conditions (11) can be re-written as

$$
B_{1}\left[\begin{array}{c}
A_{T E}^{1, n} \\
A_{T M}^{1, n}
\end{array}\right]=B_{2}\left[\begin{array}{c}
A_{T E}^{2, n-1} \\
A_{T M}^{2, n-1}
\end{array}\right] e^{-\lambda L}, \quad B_{1}\left[\begin{array}{c}
A_{T E}^{2, n} \\
A_{T M}^{2, n}
\end{array}\right]=B_{2}\left[\begin{array}{c}
A_{T E}^{1, n-1} \\
A_{T M}^{1, n-1}
\end{array}\right] e^{-\lambda L} .
$$

The convergence factor is given by the spectral radius of the iteration matrix $B:=B_{1}^{-1} B_{2} e^{-\lambda L}$, see for example [4], and this matrix is diagonal:

$$
B=\frac{\lambda-i \tilde{\omega}}{\lambda+i \tilde{\omega}}\left[\begin{array}{cc}
\frac{\tilde{\alpha}}{\alpha} & 0 \\
0 & -\frac{\tilde{\beta}}{\beta}
\end{array}\right] e^{-\lambda L} .
$$

Hence the spectral radius of $B$ is the convergence factor given in (28).

The results for 12 can be obtained similarly: we get

$$
B_{1}=(\lambda+i \tilde{\omega})\left[\begin{array}{cc}
-\frac{k_{z}}{k_{y}} \alpha & \beta \\
\alpha & \frac{k_{z}}{k_{y}} \beta
\end{array}\right], B_{2}=(\lambda-i \tilde{\omega})\left[\begin{array}{cc}
-\frac{k_{z}}{k_{y}} \tilde{\alpha} & -\tilde{\beta} \\
\tilde{\alpha} & -\frac{k_{z}}{k_{y}} \tilde{\beta}
\end{array}\right],
$$

with

$$
\begin{aligned}
& \alpha=-1-(\lambda-i \tilde{\omega})\left(\delta_{2}^{2} \lambda-i \tilde{\omega} \delta_{4}^{2}\right), \\
& \tilde{\alpha}=1+(\lambda+i \tilde{\omega})\left(\delta_{2}^{2} \lambda+i \tilde{\omega} \delta_{4}^{2}\right), \\
& \beta=-\frac{i \tilde{\omega}}{\lambda}\left(1+(\lambda-i \tilde{\omega})\left(\lambda \delta_{3}^{2}-i \tilde{\omega} \delta_{1}^{2}\right)\right), \\
& \tilde{\beta}=\frac{i \tilde{\omega}}{\lambda}\left(1+(\lambda+i \tilde{\omega})\left(\lambda \delta_{3}^{2}+i \tilde{\omega} \delta_{1}^{2}\right)\right) .
\end{aligned}
$$

Even though the iteration matrices $B_{1,2}$ from $(35)$ and $(38)$ are different, the iteration matrix $B:=B_{1}^{-1} B_{2} e^{-\lambda L}$ is of the same form shown in (37). One only has to take the coefficients $\alpha, \tilde{\alpha}, \beta, \tilde{\beta}$ from (36) for the algorithm using transmission conditions (11), and from $(39)$ for the algorithms using transmission conditions $(12)$.

\section{Optimization}

The parameters $\delta_{l}^{j}, j=1,2, l=1,2,3,4$ can be chosen to obtain rapidly converging algorithms. The following corollary is useful, since it leads to a further simplification of the convergence factor.

Corollary 1. If we denote by $\nu:=\frac{1}{|\mathbf{k}|^{2}-2 \tilde{\omega}^{2}+i \tilde{\omega}\left(s^{t e}+s^{t m}\right)}$, and we choose in the transmission condition (12)

$$
\delta_{1}^{2}=-\nu \frac{i \tilde{\omega}+s^{t e}}{i \tilde{\omega}+s^{t m}}, \quad \delta_{2}^{2}=-\nu, \quad \delta_{3}^{2}=-\nu, \quad \delta_{4}^{2}=-\nu \frac{i \tilde{\omega}+s^{t m}}{i \tilde{\omega}+s^{t e}}
$$


or in the transmission conditions (11) the corresponding values in $(28)$, the convergence factor simplifies to

$$
\rho\left(|\mathbf{k}|, \tilde{\omega}, s^{t m}, s^{t e}\right)=\left|\frac{\lambda-i \tilde{\omega}}{\lambda+i \tilde{\omega}}\right| \max \left\{\left|\frac{\lambda-s^{t e}}{\lambda+s^{t e}}\right|,\left|\frac{\lambda-s^{t m}}{\lambda+s^{t m}}\right|\right\}\left|e^{-\lambda L}\right|,
$$

where $\lambda=\sqrt{|\mathbf{k}|^{2}-\tilde{\omega}^{2}}$ and $\tilde{\omega}=\omega \sqrt{\varepsilon \mu}$.

Proof. This result can be obtained by a direct calculation.

Several choices of $s^{t e}$ and $s^{t m}$ for optimized performance are possible: in [4, case 3 , section 3.5], the authors suggest a choice depending on the use of overlap $(L>0)$ or not $(L=0)$ :

$$
s^{t m}=s^{t e}= \begin{cases}(1+i) \frac{\left(k_{+}^{2}-\tilde{\omega}^{2}\right)^{1 / 3}}{2(C h)^{1 / 3}} & L=C h>0 \\ (1+i) \sqrt{k^{\max }}\left(k_{+}^{2}-\tilde{\omega}^{2}\right)^{1 / 4} / \sqrt{2} & L=0\end{cases}
$$

where $k^{\max }=\frac{\pi p}{h}, p$ is the order of polynomial used in the trial functions, $h$ is the local mesh size at the interface, and $k_{+}$is an estimate of the closest numerical frequency just above $\tilde{\omega}$, see 4 for more details. This choice is minimizing the convergence factor (40) uniformly for all TE-TM modes in the case with overlap, and in a bounded range $\left(0, k_{\max }\right)$ in the case without overlap, and leads to a uniformly bounded contraction factor

$$
\rho \lesssim \begin{cases}1-2 C^{1 / 3}\left(k_{+}^{2}-\tilde{\omega}^{2}\right)^{1 / 6} h^{1 / 3} & L=C h>0 \\ 1-\frac{\sqrt{2}\left(k_{+}^{2}-\tilde{\omega}^{2}\right)^{1 / 4}}{\sqrt{k^{\max }}} & L=0\end{cases}
$$

It is even possible to obtain weaker dependence on the mesh parameter, namely $1-O\left(h^{1 / 4}\right)$ without overlap and $1-O\left(h^{1 / 5}\right)$ with overlap if the two subdomains do not use the same parameter, see [4, case 5, section 3.5].

With more information about the frequency content of the TE and TM modes, one can also optimize separately. For example if the high frequency cutoff $k^{\max }$ is different for TE and TM modes, we can use in the non-overlapping case

$$
\begin{aligned}
s^{t e} & =(1+i) \sqrt{k^{\max , t e}}\left(k_{+}^{2}-\tilde{\omega}^{2}\right)^{1 / 4} / \sqrt{2}, \\
s^{t m} & =(1+i) \sqrt{k^{\max , t m}}\left(k_{+}^{2}-\tilde{\omega}^{2}\right)^{1 / 4} / \sqrt{2},
\end{aligned}
$$

and a good heuristic choice for non-conforming Nedelec element discretizations, see [40, Section 4.5.1], is $k^{\max , t e}=k^{\max }, k^{\max , t m}=\frac{2}{3} k^{\max }$. If one has more a priori information about the modes that one wants to compute, and if one starts with a zero initial guess, it is possible to tune the transmission conditions to be very effective to converge rapidly for those particular modes. If for example one knows a priori that there is only one particular TE mode in the simulation, and no other ones, one can make the method converge for this particular mode in one shot. For a more detailed explanation, see [41], last paragraph of Subsection 5.1 . 


\section{Transmission conditions rewritten for easy implementation}

With the choice $\nu:=\frac{1}{|\mathbf{k}|^{2}-2 \tilde{\omega}^{2}+i \tilde{\omega}\left(s^{t e}+s^{t m}\right)}$ in Corollary 1 the parameters $\delta_{l}^{j}$ are not constants, since they contain also the Fourier parameters $|\mathbf{k}|^{2}$, and thus the implementation of the corresponding transmission conditions (11) and (12) seems not immediate. We show here for the case of the transmission conditions (12), which are well suited for variational implementations, how they can be discretized. The case of transmission conditions (11) is similar. The idea is to multiply both sides of the transmission conditions in Fourier by the symbol $|\mathbf{k}|^{2}-2 \tilde{\omega}^{2}+i \tilde{\omega}\left(s^{t e}+s^{t m}\right)$, and then to discretize the modified transmission conditions after taking the inverse Fourier transform of the resulting relations. This leads to the additional term $-\Delta_{\tau}-2 \tilde{\omega}^{2}+i \tilde{\omega}\left(s^{t e}+s^{t m}\right)$ and the modified, but equivalent transmission conditions

$$
\begin{aligned}
\tilde{\mathcal{T}}_{\mathbf{n}}^{2}(\mathbf{E})=\left(-\Delta_{\tau}-2 \tilde{\omega}^{2}+i \tilde{\omega}\left(s^{t e}+s^{t m}\right)\right)(\mathbf{n} \times(\nabla \times \mathbf{E})-i \tilde{\omega}(\mathbf{n} \times(\mathbf{E} \times \mathbf{n}))) \\
+\left(\tilde{\delta}_{1}^{2} \mathcal{S}_{T M}-\mathcal{S}_{T E}\right)(\mathbf{n} \times(\nabla \times \mathbf{E}))+i \tilde{\omega}\left(-\mathcal{S}_{T M}+\tilde{\delta}_{4}^{2} \mathcal{S}_{T E}\right)(\mathbf{n} \times(\mathbf{E} \times \mathbf{n})),
\end{aligned}
$$

where $\tilde{\delta}_{l}^{2}=\nu \delta_{l}^{2}, l=1,2,3,4$, and we have used already the choice of Corollary 1 that leads to $\tilde{\delta}_{2}^{2}=\tilde{\delta}_{3}^{2}=-1$. Using now the fact that $-\Delta_{\tau} I=-\mathcal{S}_{T M}+\mathcal{S}_{T E}$ we can further simplify to obtain

$$
\begin{aligned}
\tilde{\mathcal{T}}_{\mathbf{n}}^{2}(\mathbf{E}) & =\left(-2 \tilde{\omega}^{2}+i \tilde{\omega}\left(s^{t e}+s^{t m}\right)\right)(\mathbf{n} \times(\nabla \times \mathbf{E})-i \tilde{\omega}(\mathbf{n} \times(\mathbf{E} \times \mathbf{n}))) \\
& +\left(-\mathcal{S}_{T M}+\mathcal{S}_{T E}\right)(\mathbf{n} \times(\nabla \times \mathbf{E})-i \tilde{\omega}(\mathbf{n} \times(\mathbf{E} \times \mathbf{n}))) \\
& +\left(\delta_{1}^{2} \mathcal{S}_{T M}-\mathcal{S}_{T E}\right)(\mathbf{n} \times(\nabla \times \mathbf{E}))+i \tilde{\omega}\left(-\mathcal{S}_{T M}+\delta_{4}^{2} \mathcal{S}_{T E}\right)(\mathbf{n} \times(\mathbf{E} \times \mathbf{n})) \\
& =\left(-2 \tilde{\omega}^{2}+i \tilde{\omega}\left(s^{t e}+s^{t m}\right)\right)(\mathbf{n} \times(\nabla \times \mathbf{E})-i \tilde{\omega}(\mathbf{n} \times(\mathbf{E} \times \mathbf{n}))) \\
& +\left(\delta_{1}^{2}-1\right) \mathcal{S}_{T M}(\mathbf{n} \times(\nabla \times \mathbf{E}))+i \tilde{\omega}\left(\delta_{4}^{2}-1\right) \mathcal{S}_{T E}(\mathbf{n} \times(\mathbf{E} \times \mathbf{n}))
\end{aligned}
$$

If we divide further by the constant $-2 \tilde{\omega}^{2}+i \tilde{\omega}\left(s^{t e}+s^{t m}\right)$, the proposed transmission condition can be rewritten as

$$
\begin{aligned}
\tilde{\mathcal{T}}_{\mathbf{n}}^{2}(\mathbf{E}) & =\mathbf{n} \times(\nabla \times \mathbf{E})-i \tilde{\omega}(\mathbf{n} \times(\mathbf{E} \times \mathbf{n})) \\
& +\frac{1}{\tilde{\omega}^{2}-i \tilde{\omega} s^{t m}} \mathcal{S}_{T M}(\mathbf{n} \times(\nabla \times \mathbf{E}))+\frac{i}{\tilde{\omega}-i s^{t e}} \mathcal{S}_{T E}(\mathbf{n} \times(\mathbf{E} \times \mathbf{n})) .
\end{aligned}
$$

We see that the optimized transmission conditions (46) contain, as a first part, the classical impedance conditions $(10)$, and then, as a second part, a perturbation term using the operators $\mathcal{S}_{T M}$ and $\mathcal{S}_{T E}$. These two second order differential operators can be implemented using integration by parts in a variational framework, see for example [42], where also the discretization of cross points is discussed. If the parameters in the transmission conditions (46) are derived by minimizing the convergence factor for all TE-TM modes like in (41), we call the method obtained optimized Schwarz Method (OSM). We will see in the numerical experiments in the next section that OSMs exhibit in general faster convergence compared to using other transmission conditions, like the ones suggested in [42] and [13]. Finally, we emphasize that the optimized parameters in 41) which stemm from the analysis of the constant coefficient case with planar interfaces can also be used in a general situation with curved interfaces and variable coefficients: to do so, one implements (46) variationally, and 
uses in the parameter formulas 41 the local mesh size and coefficient values. A rigorous analysis of curved interfaces is a new area that just started, see [43] and references therein.

\section{Numerical results}

We study now the performance of the optimized Schwarz algorithms via numerical experiments. We start by introducing the notation for the domain decomposition (DD) and discretization. We then test the effectiveness of the proposed OSM transmission conditions both by examining the eigenspectrum of the DD matrix, and by numerical convergence and scalability experiments with respect to several parameters of interest. We conclude with a convergence comparison for the COBRA cavity, an important large-scale electromagnetic problem, where the new OSM transmission conditions lead to significant savings. All our simulations use non-overlapping decompositions (for some overlappig experiments, see [4]), and all computational statistics are reported using a workstation with two Intel Xeon E5-2600 processors and 96 GB of memory.

\subsection{Domain decomposition and discretization}

We decompose the computational domain $\Omega \subset \mathbb{R}^{3}$ into $M$ non-overlapping subdomains such that $\Omega=\Omega_{1} \cup \Omega_{2} \cdots \cup \Omega_{M}$. We mesh each subdomain independently with a tetrahedral mesh $\mathcal{T}_{m}^{h}$ with characteristic mesh size $h$. On each subdomain, we define discrete trial and test functions $\mathbf{u}_{m}^{h}, \mathbf{v}_{m}^{h} \in \mathbf{X}_{m}^{h} \subset$ $\mathbf{H}\left(\operatorname{curl} ; \Omega_{m}\right)$, and for all our experiments $\mathbf{X}_{m}^{h}$ is the space of mixed order curlconforming vector basis functions defined in [44], with order $p=2$ (with 20 vector basis functions within each tetrahedron).

The matrix equation resulting from the finite dimensional discretization can be written in compact form, for example for two subdomains, $M=2$, as

$$
\left[\begin{array}{ll}
\mathcal{A}_{1} & \mathcal{C}_{12} \\
\mathcal{C}_{21} & \mathcal{A}_{2}
\end{array}\right]\left[\begin{array}{l}
\mathbf{u}_{1} \\
\mathbf{u}_{2}
\end{array}\right]=\left[\begin{array}{l}
\mathbf{y}_{1} \\
\mathbf{y}_{2}
\end{array}\right]
$$

where the matrices $\mathcal{A}_{1}$ and $\mathcal{A}_{2}$ are subdomain matrices and $\mathcal{C}_{12}$ and $\mathcal{C}_{21}$ are interface coupling matrices. More details on the submatrices and right-handsides can be found in 42. We solve the linear system 47) iteratively using a preconditioned Krylov subspace method. The OSM methods correspond then to a block-Jacobi preconditioner inverting only the subdomain matrices, leading to the preconditioned linear system

$$
\left[\begin{array}{cc}
\mathcal{I} & \mathcal{A}_{1}^{-1} \mathcal{C}_{12} \\
\mathcal{A}_{2}^{-1} \mathcal{C}_{21} & \mathcal{I}
\end{array}\right]\left[\begin{array}{l}
\mathbf{u}_{1} \\
\mathbf{u}_{2}
\end{array}\right]=\left[\begin{array}{c}
\mathcal{A}_{1}^{-1} \mathbf{y}_{1} \\
\mathcal{A}_{2}^{-1} \mathbf{y}_{2}
\end{array}\right]
$$

\subsection{Eigenspectrum}

Our convergence analysis predicts the eigenvalue distribution of the preconditioned DD matrix in (48). In this subection, we investigate numerically the eigenvalue distributions that results from the OSM transmission conditions. 


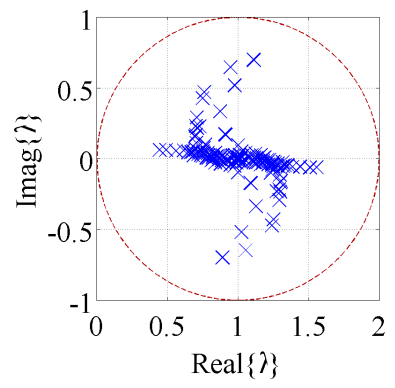

(a) RPL condition

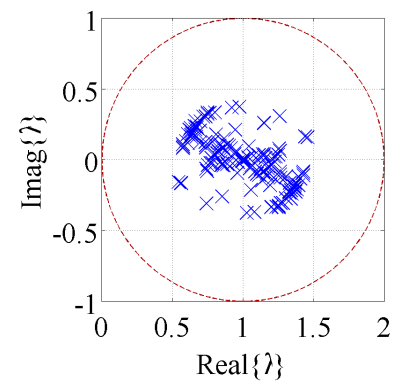

(b) OSM condition

Figure 1: Eigenspectra for a WR-75 waveguide, $f=12 \mathrm{GHz}$.

We study the concrete example of a $0.025 \mathrm{~m}$ segment of a WR-75 rectangular waveguide. The waveguide name WR stands for Waveguide Rectangular and the frequency band of operation is $\mathrm{X}-\mathrm{Ku}$ band. The dimensions of the cross-section are $0.01905 m \times 0.009595 m$. We partition the waveguide by a transverse plane into two equally sized subdomains, and use quasi-uniform meshes with mesh size $h=0.005 \mathrm{~m}$. The dimensions of the subdomains are $0.01905 m \times 0.009595 m \times 0.0125 m$.

For comparison purposes, we also show results for the transmission conditions proposed in [42] and [13, which we call here "Rawat - Peng - Lee" (RPL) transmission conditions, see also 39. The convergence factor of the RPL conditions is given by

$\rho_{R P L}\left(|\mathbf{k}|, \tilde{\omega}, \tilde{k}^{t e}, \tilde{k}^{t m}\right)=\left|\frac{\sqrt{|\mathbf{k}|^{2}-\tilde{\omega}^{2}}-i \tilde{\omega}}{\sqrt{|\mathbf{k}|^{2}-\tilde{\omega}^{2}}+i \tilde{\omega}}\right| \cdot \max \left(\left|\frac{\sqrt{|\mathbf{k}|^{2}-\tilde{\omega}^{2}}-i \tilde{k}^{t e}}{\sqrt{|\mathbf{k}|^{2}-\tilde{\omega}^{2}}+i \tilde{k}^{t e}}\right|,\left|\frac{\sqrt{|\mathbf{k}|^{2}-\tilde{\omega}^{2}}-i \tilde{k}^{t m}}{\sqrt{|\mathbf{k}|^{2}-\tilde{\omega}^{2}}+i \tilde{k}^{t m}}\right|\right)$,

where $\tilde{k}^{t m}$ and $\tilde{k}^{t e}$ are pure imaginary parameters to be chosen. The authors in [42] recommended to use

$$
\tilde{k}^{t e}=-i \sqrt{\left(\frac{1}{2}\left(k^{\max , t e}+\tilde{\omega}\right)\right)^{2}-\tilde{\omega}^{2}}, \quad \tilde{k}^{t m}=-i \sqrt{\left(\frac{1}{2}\left(k^{\max , t m}+\tilde{\omega}\right)\right)^{2}-\tilde{\omega}^{2}},
$$

see the last paragraph in Section 4 for more information on estimates for $k^{\text {max,te }}$ and $k^{\max , t m}$.

We first operate the waveguide above cutoff for only the $\mathrm{TE}_{10}$ mode, at $f=12 \mathrm{GHz}$. We show in Figure 1 the eigenvalue distributions of the DD matrices for OSM and RPL transmission conditions. We see that both spectra will lead to good convergence properties, since all the eigenvalues are within the shifted-unit-circle around 1, but the spectrum for the OSM transmission conditions is more clustered than the one for the RPL transmission conditions. This is because the OSM conditions are based on minimizing the convergence factor, which implies $\rho_{\mathrm{OSM}}^{\max }<\rho_{\mathrm{RPL}}^{\max }$, and thus a better clustering around one of the spectrum. 


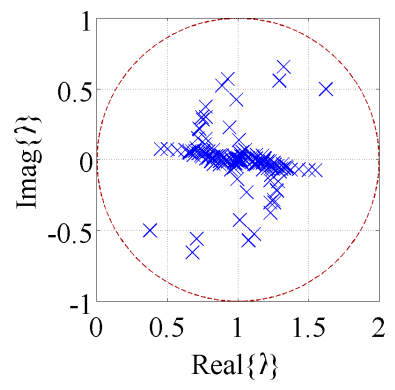

(a) RPL condition

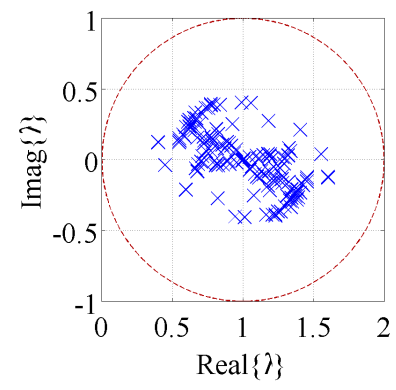

(b) OSM condition

Figure 2: EIgenspectra for a WR-75 waveguide, $f=16 \mathrm{GHz}$.

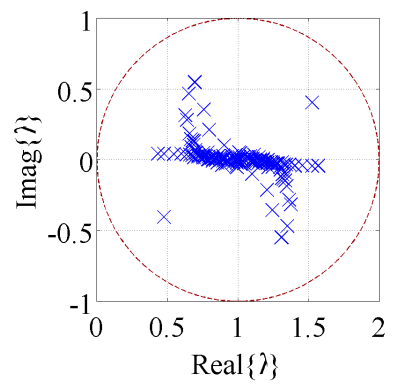

(a) RPL condition

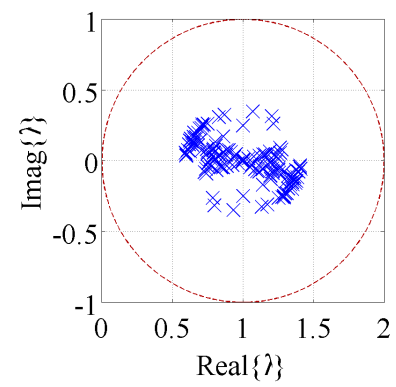

(b) OSM condition

Figure 3: EIgenspectra for a WR-75 waveguide, $f=9 \mathrm{GHz}$.

Next, we use the same discretization, $h=0.005 \mathrm{~m}$ and $p=2$, and increase the frequency to $f=16 \mathrm{GHz}$. At this operating frequency, both the $\mathrm{TE}_{10}$ and $\mathrm{TE}_{20}$ modes propagate. This is reflected in Figure 2 where we see that the spectral radii increased slightly in both cases. Again, we notice that the OSM conditions lead to a smaller spectral radius and eigenvalues more clustered around one.

We finally repeat the numerical analysis at frequency $f=9 \mathrm{GHz}$, see Figure 3. This results in more evanescent modes in the eigenspectrum, and again we observe the better clustering of the OSM conditions around one, as before, which confirms our analysis for all these regimes. We can therefore expect that the OSM transmission conditions will lead to smaller iteration numbers, at the same numerical cost per iteration, an issue we will study numerically in the next subsection.

\subsection{Convergence study}

We now use the truncated Generalized Conjugate Residual method (GCR), see [45], with the block Gauss-Seidel preconditioner corresponding to the alter- 
Table 1: Number of iterations to attain a relative residual reduction of $10^{-8}$ for different transmission conditions and different mesh sizes.

\begin{tabular}{lcccc}
\hline Cases & $\tilde{\omega} h=0.785$ & $\tilde{\omega} h=0.524$ & $\tilde{\omega} h=0.393$ & $\tilde{\omega} h=0.256$ \\
\hline RPL conditions & $11(20)$ & $14(29)$ & $17(39)$ & $19(53)$ \\
OSM conditions & $12(20)$ & $10(23)$ & $10(25)$ & $10(26)$ \\
\hline
\end{tabular}

Table 2: Computational statistics for WR-75 waveguide simulations at different mesh sizes.

\begin{tabular}{lcccc}
\hline Cases & $\tilde{\omega} h=0.785$ & $\tilde{\omega} h=0.524$ & $\tilde{\omega} h=0.393$ & $\tilde{\omega} h=0.256$ \\
\hline DOFs & 83,910 & 312,828 & 685,458 & $1,235,408$ \\
Peak memory (MB) & 28 & 98 & 182 & 329 \\
CPU time (second) & 1 & 7 & 26 & 63 \\
\hline
\end{tabular}

nating form of our Schwarz methods to solve (47). (Note that the theoretical analysis performed in the previous sections can be extended easily to this form.) The direction of the Gauss-Seidel preconditioner has chosen to be consistent with the wave front propagation in the experiments. We denote the relative residual by $\epsilon$, and terminate the iteration when the relative reduction in $\epsilon$ is smaller than a specified tolerance $\epsilon_{0}$. To present scalability results with respect to different parameters of interest, we use $\tilde{\omega}$ for the wave number, $d$ for the subdomain size, $D$ for the entire problem domain size, and $h$ for the mesh size.

\subsubsection{Scalability with respect to $\tilde{\omega} h$}

We use a $1.5 \lambda_{0}$ segment of a WR-75 rectangular waveguide operating at 12 $\mathrm{GHz}$, where $\lambda_{0}$ denotes the free space wavelength. The waveguide ports are terminated with perfectly matched layers (PMLs) and as excitation, we use the $\mathrm{TE}_{10}$ mode. We partition the waveguide into six subdomains, each $0.25 \lambda_{0}$ long. These subdomains are meshed independently and quasi-uniformly, and the interface meshes do not match. The iteration numbers required using the RPL and OSM transmission conditions for varying mesh sizes from $h=\lambda_{0} / 8$ to $h=\lambda_{0} / 20$ are given in Table1. The number of degrees of freedom (DOFs), peak memory usage and computational time per DD iteration are given in Table 2 . Note that the DD method with OSM conditions and the DD method with the RPL conditions require the same computational cost per iteration.

The $h$-refinement permits the representation of more high frequency evanescent modes on the interface. Since both RPL and OSM conditions deal effectively with evanescent modes, the dependence of the iteration count on $\tilde{\omega} h$ is small, but asymptotically more pronounced for the RPL transmission conditions than for the OSM transmission conditions, as expected from our spectral analysis. We next repeat the experiment using a random vector as the right-hand side to make sure all frequencies are present in the excitation. The corresponding results are shown in parentheses in Table 1. We see that more iterations are needed when all modes are present compared to the $\mathrm{TE}_{10}$ mode excitation, but note again the asymptotic advantage of the OSM transmission conditions when 
$\tilde{\omega} h$ becomes small.

\subsubsection{Scalability with respect to $\tilde{\omega} D$}

We use a WR-75 waveguide of length $0.0375 \mathrm{~m}$ partitioned into 6 equally sized subdomains of dimension $0.01905 \mathrm{~m} \times 0.009595 \mathrm{~m} \times 0.00625 \mathrm{~m}$. We test the performance of the DD methods for four frequencies: $12 \mathrm{GHz}, 20 \mathrm{GHz}, 30$ $\mathrm{GHz}$ and $40 \mathrm{GHz}$. The electrical size of the waveguide increases from $0.76 \lambda_{0} \times$ $0.38 \lambda_{0} \times 1.5 \lambda_{0}$ to $2.54 \lambda_{0} \times 1.28 \lambda_{0} \times 5 \lambda_{0}$ accordingly. The mesh size is chosen as $h=\lambda_{0} / 8$. The iteration counts we obtain are given in Table 3 for both $\mathrm{TE}_{10}$ mode excitation and in parentheses with a random vector as excitation. The computational statistics for the simulations are given in Table 4. We see that the DD method with OSM transmission conditions depends only little on $\tilde{\omega} D$, and it clearly outperforms the RPL transmission conditions when the operating frequency increases.

We next examine the behavior of the methods as the problem size increases. We use a fixed subdomain size of $0.25 \lambda_{0}$, and we increase the length of the waveguide by increasing the number of subdomains. The mesh size is kept fixed at $h=\lambda_{0} / 8$. Figure 4 shows the convergence history of the methods for 10, 40, 160, 640 subdomains, where the electrical size of the problems increases from $2.5 \lambda_{0}$ to $160 \lambda_{0}$ accordingly. Table 5 gives the computational statistics of the simulations. Note that we have exploited the subdomain repetitions in the computation [46], so the computational resources required are quite modest.

In this convergence study, the propagating modes are of great significance because the wave must travel from one end of the waveguide to the other. We see that both RTL and OSM transmission conditions lead to a dependence of the convergence on the problem size, which is expected in the absence of a coarse space. However, the OSM conditions significantly outperform the RTL conditions. In the top row of Figure 4 we show the results for a $\mathrm{TE}_{10}$ mode excitation, and below for a random vector excitations, where the dependence on the number of subdomains with OSM transmission conditions is only very moderate.

\subsection{Electromagnetic scattering from a COBRA cavity}

The second example we consider is the electromagnetic wave scattering from a COBRA cavity, whose geometrical description is given in Figure 5 We consider a plane wave normal incident upon the cavity aperture, $\theta=180^{\circ}$, $\phi=90^{\circ}$, at frequency of $f=10 \mathrm{GHz}$. The electric field is polarized in the $\hat{\mathbf{x}}$ direction. The cavity is partitioned into 4 repeated subblocks and meshed with

Table 3: Number of iterations to attain a relative residual reduction of $10^{-8}$ for different transmission conditions and different operating frequencies

\begin{tabular}{lcccc}
\hline Cases & $f=12 \mathrm{GHz}$ & $f=20 \mathrm{GHz}$ & $f=30 \mathrm{GHz}$ & $f=40 \mathrm{GHz}$ \\
\hline RPL conditions & $11(20)$ & $15(31)$ & $19(43)$ & $32(59)$ \\
OSM conditions & $12(20)$ & $14(23)$ & $15(25)$ & $17(27)$ \\
\hline
\end{tabular}




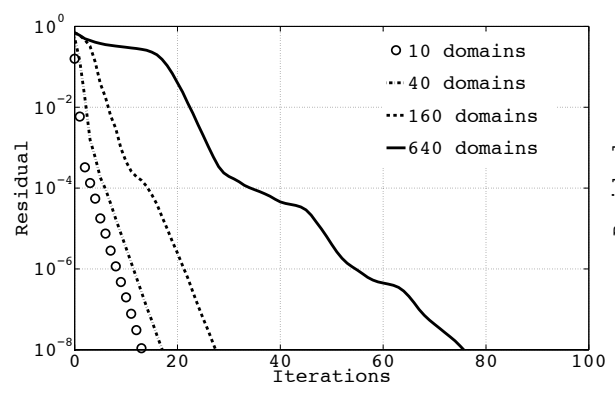

(a) RPL conditions

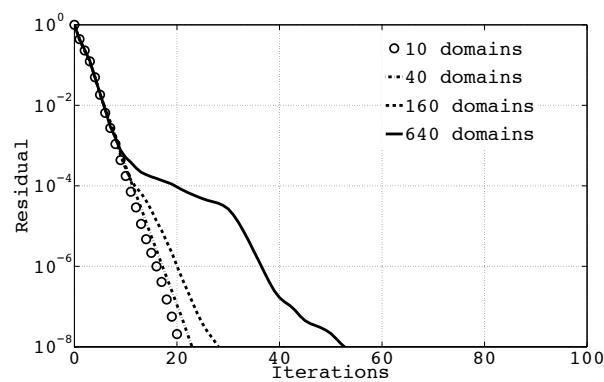

(c) RPL conditions

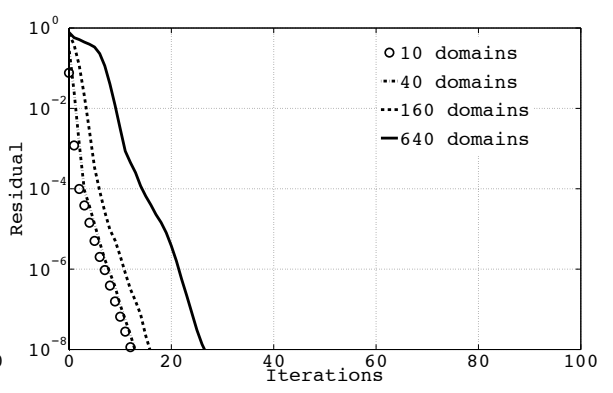

(b) OSM conditions

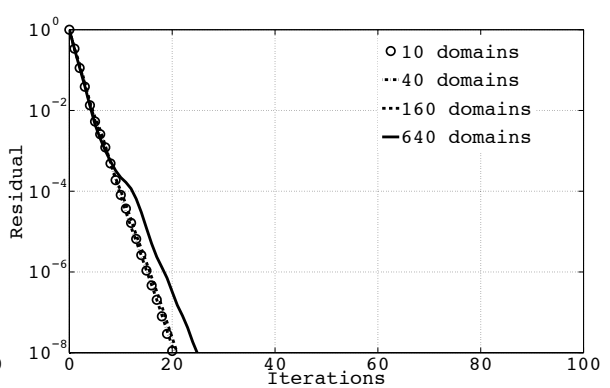

(d) OSM conditions

Figure 4: Iterative solver convergence with increased problem size, $\mathrm{TE}_{10}$ mode excitation in the top row, and random vector excitation in the bottom row.

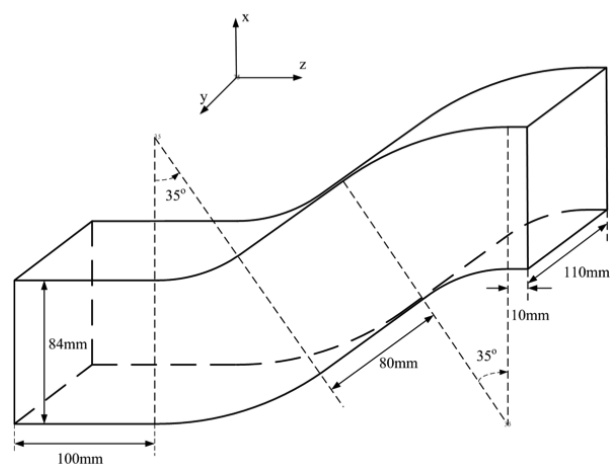

(a) Geometry

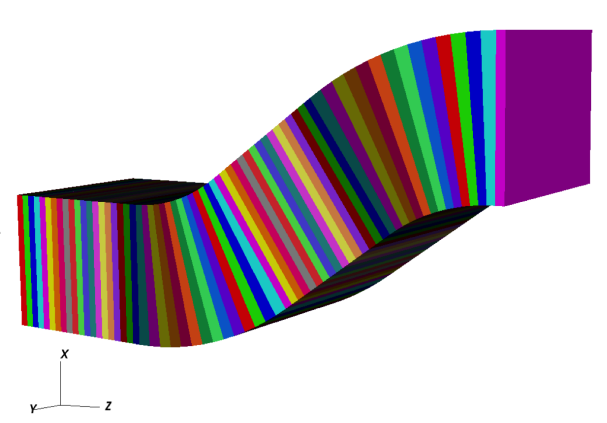

(b) Domain Partition

Figure 5: Decomposition of the COBRA cavity into 65 subdomains. 
mesh size $h=\lambda_{0} / 8$. We use 65 subdomains arranged using these subblocks as shown in Figure 5, leading to 23,564,846 degrees of freedom. The peak memory usage is $12 \mathrm{~GB}$ and it takes 30 minutes for one DD iteration.

Figure 6 shows the convergence history of the methods. The DD method with OSM transmission conditions requires 69 iterations to reach $\epsilon_{0}=10^{-8}$. The DD method with RPL transmission conditions fails to converge to $\epsilon_{0}=$ $10^{-8}$ within 300 iterations. Next, we perform a simulation using an oblique incidence, $\theta=150^{\circ}, \phi=90^{\circ}$, and an $\hat{\mathbf{x}}$-polarized electric field. The simulation with oblique incidence requires 81 iterations using OSM transmission conditions, compared to more than 300 iterations with RPL transmission conditions, again a significant improvement. The electric fields on the COBRA cavity are shown in Figure 7 for both the normal and oblique excitations.

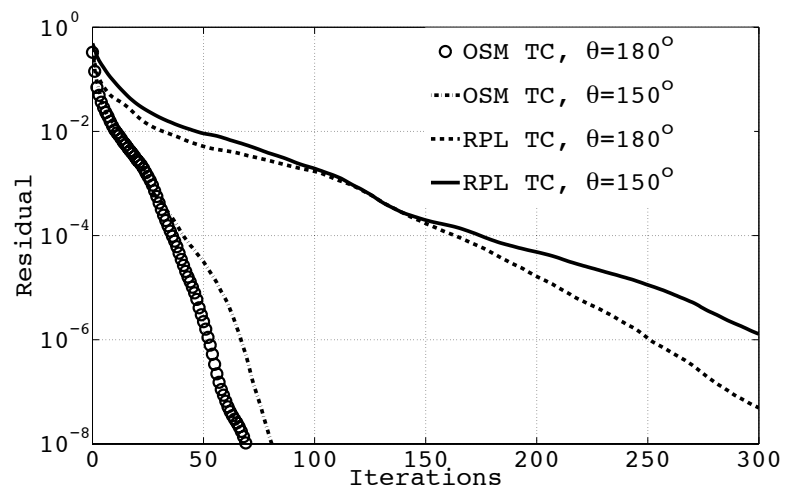

Figure 6: Iterative solver convergence for the COBRA cavity

\subsection{Electromagnetic radiation from a Vivaldi antenna array}

We conclude our numerical experiments with a large-scale electromagnetic radiation problem. Shown in Figure 8 is a mockup nose radar antenna array often placed on the front of the airplane behind the radome. It is an X-band Vivaldi-type antenna array [47] with total 864 antenna elements, and distributed on a planar grid. Each antenna element is considered as one subdomain and discretized using adaptive $h$ mesh refinement [4, which results in average mesh size $h=\lambda_{0} / 12$ for the computation. The exterior mesh truncation boundary is enforced by a combined field boundary integral equation method [49]. The

Table 4: Computational statistics for the WR-75 waveguide simulations at different operating frequencies.

\begin{tabular}{lcccc}
\hline Cases & $f=12 \mathrm{GHz}$ & $f=20 \mathrm{GHz}$ & $f=30 \mathrm{GHz}$ & $f=40 \mathrm{GHz}$ \\
\hline DOFs & 83,910 & 675,936 & $2,151,138$ & $4,761,927$ \\
Peak memory (MB) & 28 & 175 & 512 & 1,200 \\
CPU time (mm:ss) & $00: 01$ & $00: 20$ & $02: 00$ & $05: 00$ \\
\hline
\end{tabular}




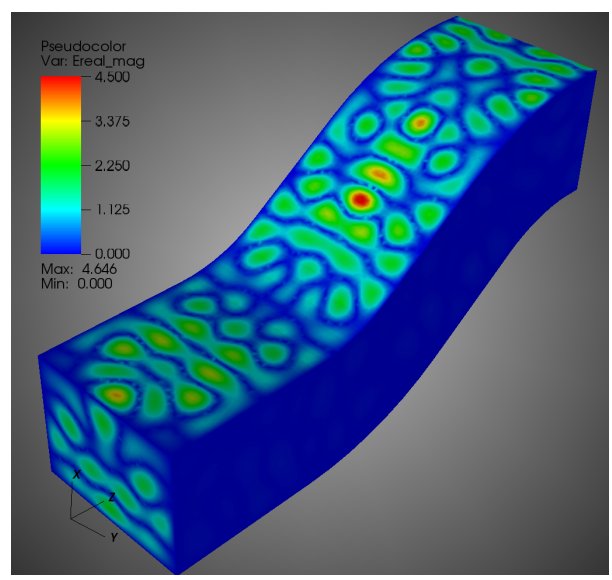

(a) Normal incident excitation: $\theta=180^{\circ}, \phi=$ $90^{\circ}$

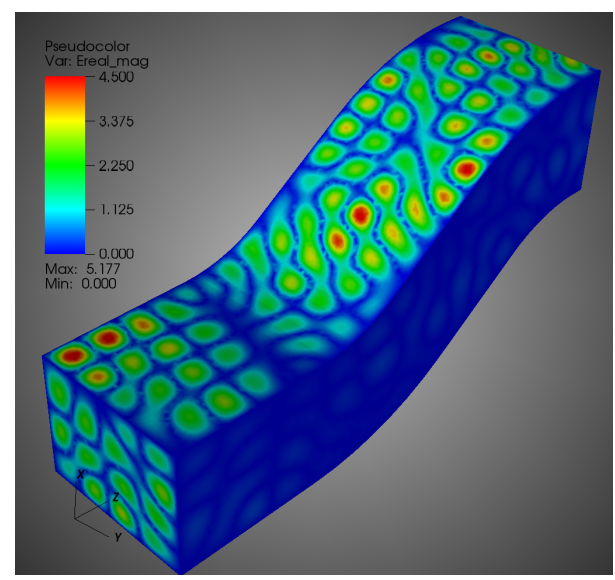

(b) Obliquely incident excitation: $\theta=150^{\circ}, \phi=$ $90^{\circ}$

Figure 7: Electric field magnitude on the COBRA cavity.

simulation is performed at $10 \mathrm{GHz}$ with 864 subdomains and requires $26,541,216$ DOFs. It requires 7 minutes for one DD iteration and peak memory usage is $5.8 \mathrm{~GB}$. The convergence history of the DD methods with RPL transmission conditions and OSM transmission conditions are shown in Figure 9, The electric current distribution on the antenna array and the radiation magnetic field on a observation surface are shown in Figure 10.

\section{Conclusions}

We explained in this paper how many transmission conditions for solving time-harmonic Maxwell equations by domain decomposition can be formulated and analyzed in the common framework of optimized Schwarz methods. In particular, using the important TE-TM decomposition, we derived explicit formulas which allow us to use optimized transmission conditions developed for first order formulations directly also for second order formulations, for which such optimized formulas were missing so far. Numerical experiments showed that these optimized transmission conditions lead to domain decomposition methods

Table 5: Computational statistics for the WR-75 waveguide simulations with increasing problem size ( $N$ : number of subdomains).

\begin{tabular}{lcccc}
\hline Cases & $N=10$ & $N=40$ & $N=160$ & $N=640$ \\
\hline DOFs & 136,902 & 534,342 & $2,124,102$ & $8,483,142$ \\
Peak memory (MB) & 22 & 65 & 265 & 1,067 \\
CPU time (second) & 0.5 & 2 & 8 & 33 \\
\hline
\end{tabular}



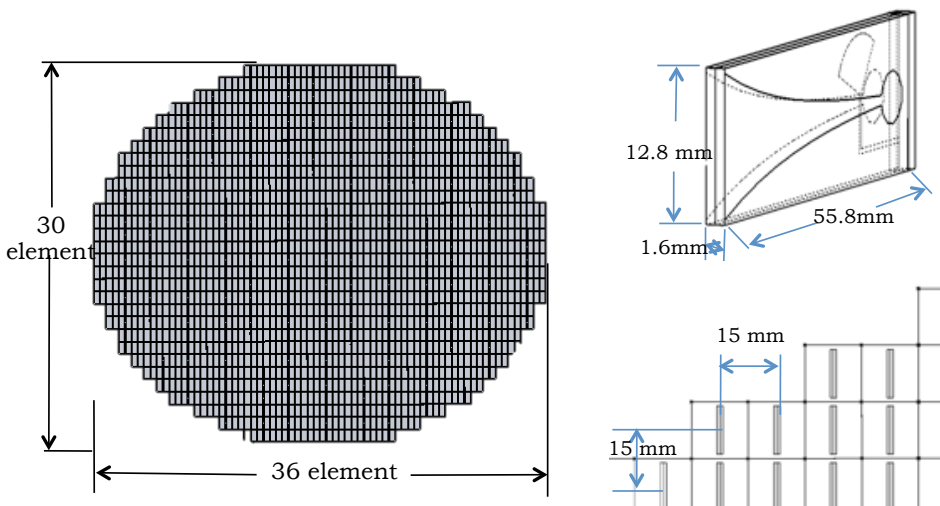

Total number of elements: 864

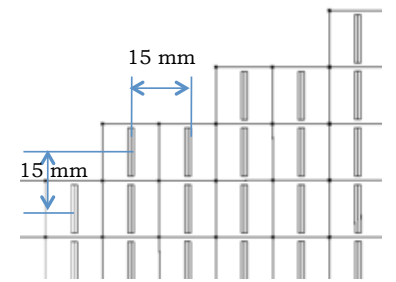

Figure 8: Configuration and geometry description of a Vivaldi antenna array

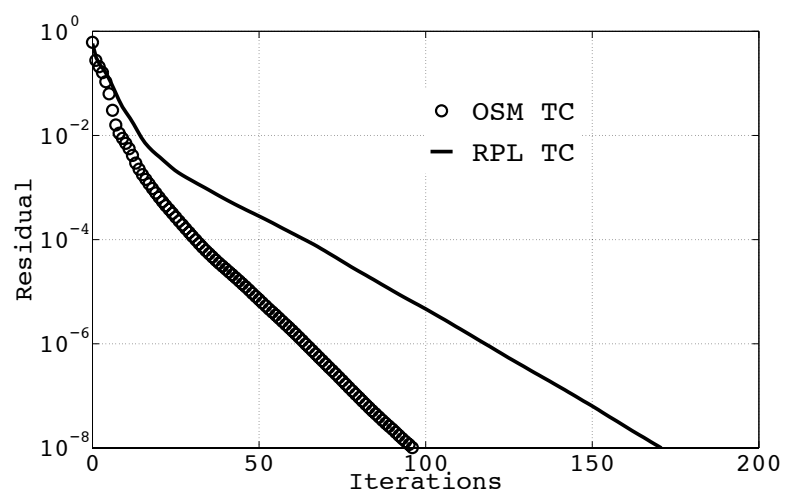

Figure 9: Iterative solver convergence for the Vivaldi antenna array

for the second order time-harmonic Maxwell equations that scale well with respect to the discretization size, the operating frequency and the problem size, and outperform existing transmission conditions. Finally, we demonstrated substantially improved convergence for an important large scale simulation, at the same cost per iteration.

\section{References}

[1] Y. Courvoisier, M. Gander, Time domain Maxwells equations and Schwarz waveform relaxation methods, in: Domain Decomposition Methods in Science and Engineering XX, Springer LNCSE, 2012.

[2] V. Dolean, S. Lanteri, R. Perrussel, A domain decomposition method for solving the three-dimensional time-harmonic Maxwell equations discretized 


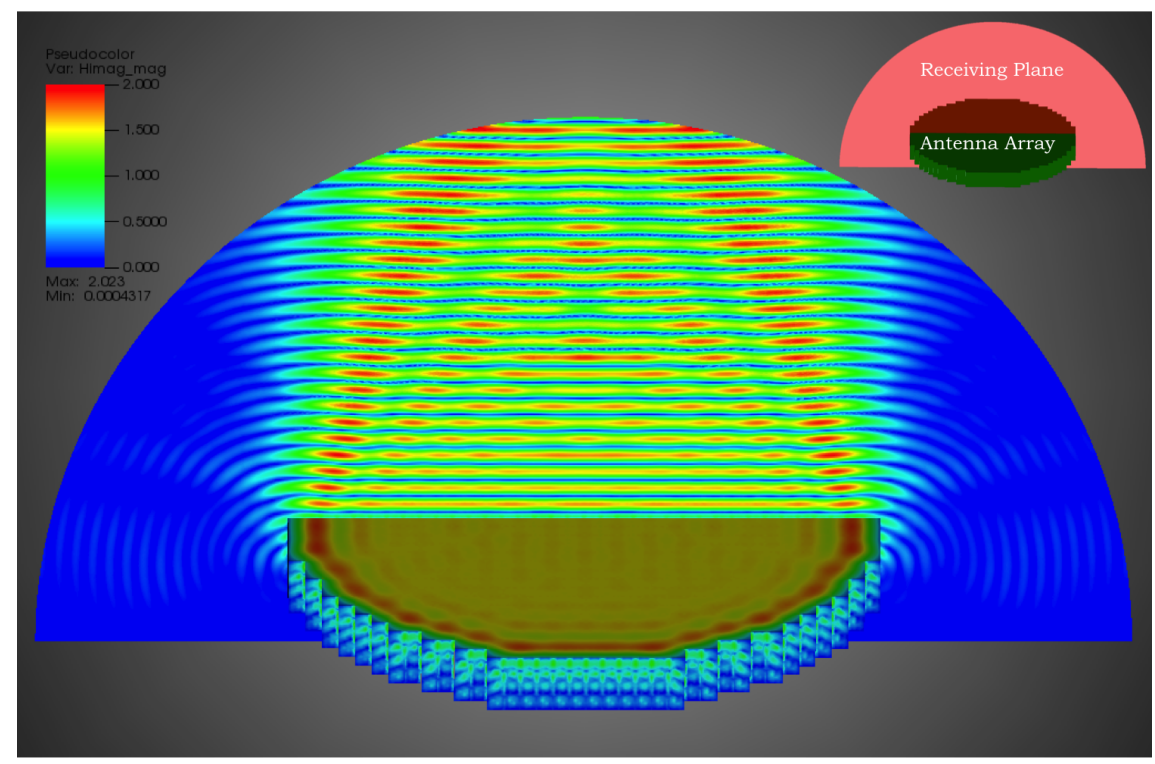

Figure 10: The electric current on the antenna array with radiated fields on a receiving plane

by discontinuous Galerkin methods, J. Comput. Phys. 227 (3) (2008) 20442072 .

[3] V. Dolean, S. Lanteri, R. Perrussel, Optimized Schwarz algorithms for solving time-harmonic Maxwell's equations discretized by a discontinuous Galerkin method, IEEE. Trans. Magn. 44 (6) (2008) 954-957.

[4] V. Dolean, L. Gerardo-Giorda, M. J. Gander, Optimized Schwarz methods for Maxwell equations, SIAM J. Scient. Comp. 31 (3) (2009) 2193-2213.

[5] M. El Bouajaji, V. Dolean, M. J. Gander, S. Lanteri, Comparison of a one and two parameter family of transmission conditions for Maxwells equations with damping, in: Domain Decomposition Methods in Science and Engineering XX, Springer LNCSE, 2012, accepted for publication.

[6] M. El Bouajaji, V. Dolean, M. J. Gander, S. Lanteri, Optimized Schwarz methods for the time-harmonic Maxwell equations with dampimg, SIAM J. Scient. Comp. 34 (4) (2012) 2048-2071.

[7] B. Després, P. Joly, J. E. Roberts, A domain decomposition method for the harmonic Maxwell equations, in: Iterative methods in linear algebra (Brussels, 1991), North-Holland, Amsterdam, 1992, pp. 475-484.

[8] P. Chevalier, Méthodes numériques pour les tubes hyperfréquences. résolution par décomposition de domaine, Ph.D. thesis, Université Paris VI (1998). 
[9] P. Collino, G. Delbue, P. Joly, A. Piacentini, A new interface condition in the non-overlapping domain decomposition, Comput. Methods Appl. Mech. Engrg. 148 (1997) 195-207.

[10] A. Alonso-Rodriguez, L. Gerardo-Giorda, New nonoverlapping domain decomposition methods for the harmonic Maxwell system, SIAM J. Sci. Comput. 28 (1) (2006) 102-122.

[11] Z. Peng, V. Rawat, J.-F. Lee, One way domain decomposition method with second order transmission conditions for solving electromagnetic wave problems, J. Comput. Phys. 229 (4) (2010) 1181-1197.

[12] Z. Peng, J.-F. Lee, Non-conformal domain decomposition method with second-order transmission conditions for time-harmonic electromagnetics, J. Comput. Phys. 229 (16) (2010) 5615-5629.

[13] V. Rawat, J.-F. Lee, Nonoverlapping domain decomposition with second order transmission condition for the time-harmonic Maxwell's equations, SIAM J. Sci. Comput. 32 (6) (2010) 3584-3603.

[14] F. Ihlenburg, I. Babuška, Finite element solution to the Helmholtz equation with high wave number. Part I: The $h$-version of the FEM, Computer Methods in Applied Mechanics and Engineering 39 (1995) 9-37.

[15] F. Ihlenburg, I. Babuška, Finite element solution to the Helmholtz equation with high wave number. Part II: The $h-p$ version of the FEM, SIAM Journal on Numerical Analysis 34 (1997) 315-358.

[16] O. Ernst, M. Gander, Why it is difficult to solve Helmholtz problems with classical iterative methods, in: I. Graham, T. Hou, O. Lakkis, R. Scheichl (Eds.), Numerical Analysis of Multiscale Problems, Springer Verlag, 2012, pp. 325-363.

[17] H. Helmholtz, Theorie der Luftschwingungen in Röhren mit offenen Enden, Journal für reine und angewandte Mathematik 57 (1859) 1-72.

[18] M. Gander, Iterative methods for Helmholtz and Maxwell equations, Mathematisches Forschungsinstitut Oberwolfach, Report 55 (2012) 10-11.

[19] A. Toselli, Some results on overlapping Schwarz methods for the Helmholtz equation employing perfectly matched layers, Tech. Rep. 765, Courant Institute, New York (June 1998).

[20] A. Toselli, Overlapping Schwarz methods for Maxwell's equations in three dimensions, Numer. Math. 86 (4) (2000) 733-752.

[21] A. Toselli, A. Klawonn, A FETI domain decomposition method for edge element approximations in two dimensions with discontinuous coefficients, SIAM J. Numer. Anal. 39 (3) (2001) 932-956. 
[22] F. Rapetti, A. Toselli, A FETI preconditioner for two-dimensional edge element approximations of Maxwell's equations on nonmatching grids, SIAM J. Sci. Comput. 23 (1) (2001) 92-108.

[23] A. Toselli, O. B. Widlund, B. I. Wohlmuth, An iterative substructuring method for Maxwell's equations in two dimensions, Math. Comp. 70 (235) (2001) 935-949.

[24] Q. Hu, J. Zou, A nonoverlapping domain decomposition method for Maxwell's equations in three dimensions, SIAM J. Numer. Anal. 41 (5) (2003) 1682-1708.

[25] R. Hiptmair, Multigrid method for Maxwell's equations, SIAM Journal on Numerical Analysis 36 (1) (1998) 204-225.

[26] R. Hiptmair, J. Xu, Nodal auxiliary space preconditioning in $\mathrm{H}(\mathrm{curl})$ and H(div) spaces, SIAM Journal on Numerical Analysis 45 (6) (2007) 24832509 .

[27] P.-L. Lions, On the Schwarz alternating method. III: a variant for nonoverlapping subdomains, in: T. F. Chan, R. Glowinski, J. Périaux, O. Widlund (Eds.), Third International Symposium on Domain Decomposition Methods for Partial Differential Equations, held in Houston, Texas, March 20-22, 1989, SIAM, Philadelphia, PA, 1990.

[28] B. Després, Décomposition de domaine et problème de Helmholtz, C.R. Acad. Sci. Paris 1 (6) (1990) 313-316.

[29] B. Després, P. Joly, J. E. Roberts, A domain decomposition method for the harmonic Maxwell equations, in: Iterative methods in linear algebra (Brussels, 1991), North-Holland, Amsterdam, 1992, pp. 475-484.

[30] M. J. Gander, Optimized Schwarz methods, SIAM J. Numer. Anal. 44 (2) (2006) 699-731.

[31] P. Chevalier, F. Nataf, Symmetrized method with optimized second-order conditions for the Helmholtz equation, in: Domain decomposition methods, 10 (Boulder, CO, 1997), Amer. Math. Soc., Providence, RI, 1998, pp. 400407.

[32] M. J. Gander, F. Magoulès, F. Nataf, Optimized Schwarz methods without overlap for the Helmholtz equation, SIAM J. Sci. Comput. 24 (1) (2002) $38-60$.

[33] P. Collino, G. Delbue, P. Joly, A. Piacentini, A new interface condition in the non-overlapping domain decomposition for the Maxwell equations Helmholtz equation and related optimal control, Comput. Methods Appl. Mech. Engrg 148 (1997) 195-207. 
[34] X. Antoine, Y. Boubendir, C. Geuzaine, A quasi-optimal non-overlapping domain decomposition algorithm for the Helmholtz equation, Journal of Computational Physic 231 (2) (2012) 262-280.

[35] M. El Bouajaji, V. Dolean, M. J. Gander, S. Lanteri, R. Perrussel, Domain decomposition methods for electromagnetic wave propagation problems in heterogeneous media and complex domains, in: Domain Decomposition Methods in Science and Engineering XIX, Vol. 78(1), Springer LNCSE, 2011, pp. 5-16.

[36] S.-C. Lee, M. Vouvakis, J.-F. Lee, A non-overlapping domain decomposition method with non-matching grids for modeling large finite antenna arrays, J. Comput. Phys. 203 (1) (2005) 1-21.

[37] J.-C. Nédélec, Acoustic and electromagnetic equations. Integral representations for harmonic problems., Applied Mathematical Sciences, 144, Springer Verlag, 2001.

[38] A. Buffa, M. Costabel, D. Sheen, On traces for $\mathrm{H}(\operatorname{curl}, \Omega)$ in Lipschitz domains, Journal of Mathematical Analysis and Applications 276 (2) (2002) 845-867.

[39] V. Dolean, M. J. Gander, S. Lanteri, J.-F. Lee, Z. Peng, Optimized Schwarz methods for curl-curl time-harmonic Maxwell's equations, in: J. Erhel, M. J. Gander, L. Halpern, T. Sassi, O. Widlund (Eds.), Proceedings of the 21st international domain decomposition conference, Springer LNCSE, 2013.

[40] V. Rawat, Finite element domain decomposition with second order transmission conditions for time-harmonic electromagnetic problems, Ph.D. thesis, Ohio State University (2009).

[41] M. J. Gander, Schwarz methods over the course of time, Electronic Transactions on Numerical Analysis 31 (2008) 228-255.

[42] Z. Peng, J.-F. Lee, A scalable nonoverlapping and nonconformal domain decomposition method for solving time-harmonic maxwell equations in $\mathbf{r}^{3}$, SIAM Journal on Scientific Computing 34 (3) (2012) A1266-A1295.

[43] M. J. Gander, Y. Xu, Optimized Schwarz methods for circular domain decompositions with overlap, SIAM J. Numer. Anal.To appear.

[44] D. K. Sun, J.-F. Lee, Z. Cendes, Construction of nearly orthogonal Nedelec bases for rapid convergence with multilevel preconditioned solvers, SIAM Journal on Scientific Computing 23 (2001) 1053-1076.

[45] S. Eisenstat, H. Elman, M. Schultz, Variational iterative methods for nonsymmetric systems of linear equations, SIAM J. Numer. Anal. 20 (2) (1983) $345-357$. 
[46] K. Zhao, V. Rawat, S.-C. Lee, J. F. Lee, A domain decomposition method with nonconformal meshes for finite periodic and semi-periodic structures, IEEE Trans. Antennas and Propagation 55 (9) (2007) 2559-2570.

[47] T. H. Chio, D. H. Schaubert, Parameter study and design of wide-band widescan dual-polarized tapered slot antenna arrays, IEEE Trans. Antennas and Propagation 48 (6) (2000) 879-886.

[48] D. K. Sun, Z. Cendes, J.-F. Lee, Adaptive mesh refinement, h-version, for solving multipart microwave devices in three dimensions, IEEE Transactions on Magnetics 4 (2000) 1596-1599.

[49] Z. Peng, K.-H. Lim, J.-F. Lee, Computations of electromagnetic wave scattering from penetrable composite targets using a surface integral equation method with multiple traces, IEEE Transactions on Antennas and Propagation 61 (1) (2013) 256-270. 